\title{
The clinical impact of breast scintigraphy acquired with a breast specific $\gamma$-camera (BSGC) in the diagnosis of breast cancer: Incremental value versus mammography
}

\author{
ANGELA SPANU $^{1}$, DANIELA SANNA ${ }^{1}$, FRANCESCA CHESSA ${ }^{1}$, ALESSANDRA MANCA ${ }^{2}$, \\ PIERINA COTTU ${ }^{3}$, ALESSANDRO FANCELLU $^{3}$, SUSANNA NUVOLI ${ }^{1}$ and GIUSEPPE MADEDDU ${ }^{1}$ \\ ${ }^{1}$ Unit of Nuclear Medicine, University of Sassari, Viale San Pietro 8; ${ }^{2}$ Unit of Pathology, University of Sassari, \\ Via Matteotti 58; ${ }^{3}$ Unit of Surgery, University of Sassari, Viale San Pietro 43, Sassari, Italy
}

Received November 12, 2011; Accepted January 16, 2012

DOI: $10.3892 /$ ijo.2012.1495

\begin{abstract}
We investigated the clinical impact of breast scintigraphy acquired with a breast specific $\gamma$-camera (BSGC) in the diagnosis of breast cancer (BC) and assessed its incremental value over mammography (Mx). A consecutive series of 467 patients underwent BSGC scintigraphy for different indications: suspicious lesions on physical examination and/or on US/MRI negative at Mx (BI-RADS 1 or 3), characterization of lesions suspicious at Mx (BI-RADS 4), preoperative staging in lesions highly suggestive of malignancy at Mx (BI-RADS 5). Definitive histopathological findings were obtained in all cases after scintigraphy: 420/467 patients had BC, while 47/467 patients had benign lesions. The scintigraphic data were correlated to Mx BI-RADS category findings and to histology. The incremental value of scintigraphy over $\mathrm{Mx}$ was calculated. Scintigraphy was true-positive in $97.1 \%$ BC patients, detecting $96.2 \%$ of overall tumor foci, including $91.5 \%$ of carcinomas $\leq 10 \mathrm{~mm}$, and it was true-negative in $85.1 \%$ of patients with benign lesions. Scintigraphy gave an additional value over $\mathrm{Mx}$ in 141/467 cases (30.2\%). In particular, scintigraphy ascertained $\mathrm{BC}$ missed at Mx in 31 patients with BI-RADS 1 or 3, including 26 patients with heterogeneously/high dense breast (19/26 with tumors $\leq 10 \mathrm{~mm}$ ) and detected additional clinically occult ipsilateral or controlateral tumor foci (all $<10 \mathrm{~mm}$ ) or the in situ component sited around invasive tumors in $77 \mathrm{BC}$ patients with BI-RADS 4 or 5 , changing surgical management in $18.2 \%$ of these cases; moreover, scintigraphy ruled out malignancy in 33 patients with BI-RADS 4 . BSGC scintigraphy proved a highly sensitive diagnostic tool, even in small size carcinoma detection, while maintaining a high specificity. The procedure increased both the sensitivity of Mx, especially in dense breast and in
\end{abstract}

Correspondence to: Dr Angela Spanu, Unit of Nuclear Medicine, University of Sassari, Viale San Pietro 8, I-07100 Sassari, Italy E-mail: angela.spanu@email.it

Key words: breast scintigraphy, breast cancer, breast specific $\gamma$-camera, mammography multifocal/multicentric disease, and the specificity as well as it better defined local tumor extension, thus guiding the surgeon to a more appropriate surgical treatment.

\section{Introduction}

As it is well known, mammography (Mx) represents the imaging procedure of reference in both the screening and the diagnosis of primary breast cancer, in spite of some limitations in sensitivity and specificity.

Mx performance is especially limited in patients with extremely high dense parenchyma, with sensitivity values dropping to approximately $50 \%$ (1), as well as in the assessment of both multifocal/multicentric and contralateral synchronous breast cancers, as demonstrated at detailed serial slicing of the mastectomy specimen and at random biopsy as well as at prophylactic mastectomy of the contralateral breast $(2,3)$.

Another important limitation of $\mathrm{Mx}$ is its intrinsic low specificity, with positive predictive values reported in a very wide range (2-95\%) for lesions with mammographic findings belonging to BI-RADS category 4 , a category for which biopsy is indicated, leading to numerous unnecessary biopsies. To overcome these limitations, since the 1990s scintimammography with $\gamma$-emitting technetium labelled radiotracers has been proposed as a complementary tool to $\mathrm{Mx}$.

However, during the course of the years, the role of scintimammography has been progressively reduced given the low sensitivity (generally $<50 \%$ ) demonstrated in the identification of small size $(<1 \mathrm{~cm})$ carcinomas (4-6), making the procedure unreliable in both the screening and the diagnosis of tumors at a very early stage.

At present, a new interest on the employment of breast radioisotopic imaging is registering due to the development of small field of view breast specific $\gamma$-cameras (BSGC) equipped with special devices which present several physical and technical advantages in respect of the general purpose $\gamma$-camera generally employed in scintimammography, such as higher spatial resolution, higher manoeuvrability, smaller lesion-to-detector distance, exclusion of nearby organs from the field of view and reduced radiation scattering in breast imaging. Moreover, dedicated devices may be inserted in a mammography gantry thus 
permitting breast imaging views comparable to those of $\mathrm{Mx}$ and a precise information on lesion site.

In confirmation of the renovated interested on radioisotopic breast imaging, practice guidelines for breast scintigraphy with BSGC have been recently published (7). This new procedure, using either ${ }^{99 \mathrm{~m}} \mathrm{Tc}$-sestaMIBI or ${ }^{99 \mathrm{~m}} \mathrm{Tc}$-tetrofosmin as radiotracer, has already demonstrated in clinical studies a high sensitivity in the detection of small size $(\leq 10 \mathrm{~mm})$ carcinomas, with values ranging from 86 to $91 \%$ (8-10) which result markedly higher that those generally achieved by conventional planar scintimammography.

In the present study we investigated the clinical impact of breast scintigraphy acquired with a BSGC in the diagnosis of primary breast cancer and we also evaluated its additional value over $\mathrm{Mx}$, in particular in the detection of small size carcinomas.

\section{Patients and methods}

Patients. We studied prospectively 467 consecutive female patients, aged 26-81 years (median age 57 years), with breast lesions at physical examination and/or at diagnostic imaging procedures (mammography and/or breast ultrasound or MRI). The different indications were: suspicious lesions on physical examination and/or on breast ultrasonography or MRI negative at Mx (BI-RADS 1 or 3; 41 cases), characterization of lesions suspicious at Mx (BI-RADS 4; 147 cases), preoperative local staging of lesions highly suggestive of malignancy at Mx (BI-RADS 5; 279 cases).

Ten of the patients had already undergone contralateral quadrantectomy $(n=6)$ or mastectomy $(n=4)$ for infiltrating ductal carcinoma 2-12 years previously. All patients, at the time of our observation had already undergone both clinical examination and mammography.

The clinical examination had been performed in all cases by an experienced clinician. Palpable breast lumps, skin thickening or retraction, and nipple discharge or retraction were noted.

Mammographic examination included routine cranio-caudal (CC) and medio-lateral oblique (MLO) views of the breasts and at least another projection or magnification view over the area of suspected lesions. The lesions were described according to the American College of Radiology Breast Imaging Reporting and Data System (BI-RADS) Lexicon (11) for which the mammographic findings were classified as normal (BI-RADS category 1), benign (BI-RADS category 2), probably benign (BI-RADS category 3), suspicious (BI-RADS category 4) or highly suggestive of malignancy (BI-RADS category 5). In our series, 38/467 patients were classified as BI-RADS category 1, 3/467 as BI-RADS category 3, 147/467 as BI-RADS category 4 and 279/467 as BI-RADS category 5. Categories 1-3 were considered negative and categories 4-5 were considered positive. All the 41 patients negative at Mx (BI-RADS categories 1 and 3) had unclear lesions at physical examination and/or at breast ultrasonography or MRI.

The final diagnosis was obtained in all 467 patients within 2 weeks of scintigraphy by surgical (52 cases) or by percutaneous (415 cases) biopsy. Patients with proven-biopsy primary breast cancer had surgery at the same surgical department and the operation was planned according to the data derived from conventional diagnostic and scintigraphic procedures. At our institution, patients with a unifocal carcinoma $<3 \mathrm{~cm}$ usually undergo conservation surgery, while those with larger carcinomas or with multicentric carcinomas are submitted to mastectomy. Patient's opinion on the type of surgery is considered in all cases, also including the option of contralateral prophylactic mastectomy.

Patients with locally advanced primary breast cancer considered eligible for neoadjuvant chemotherapy and patients who had undergone surgical excisional biopsy were excluded from the present study.

According to the final histopathological findings, 420/467 patients had a primary breast cancer, bilateral in $8 / 420$ cases and multifocal/multicentric in 48/420 cases, while the remaining 47/467 patients had a benign disease, bilateral in one case; a benign lesion was also ascertained in 7 breast cancer patients. Table I reports the histological diagnosis of the 467 patients enrolled in the study, while the patologic $\mathrm{T}$ stage of the 420 breast cancer patients is reported in Table II. In total, 554 breast lesions were ascertained at histology: 499 tumor foci, 453 of which invasive (142 $\leq 10 \mathrm{~mm}$ and $311>10 \mathrm{~mm})$ and 46 in situ, and 55 benign lesions. Two hundred and sixty-two/420 breast cancer patients were treated with conservation surgery, while $158 / 420$ patients had mastectomy. Moreover, contralateral prophylactic mastectomy was performed in 3 breast cancer patients.

Breast specific $\gamma$-camera (BSGC) scintigraphy. BSGC scintigraphy was acquired starting $10 \mathrm{~min}$ after the intravenously injection of $740 \mathrm{MBq}$ of ${ }^{99 \mathrm{~m}} \mathrm{Tc}$-tetrofosmin (Myoview, Amersham Health - GE Healthcare) in the arm contralateral to the affected breast. In patients with bilateral breast lesions, the injection was performed in a pedal vein. Radiolabelling and quality control procedures of the radiotracer were carried out according to the manufacturer's instructions. Labelling efficiency was always $>95 \%$. Scintigraphic images were acquired at the peak of technetium $(140 \mathrm{Kev})$ with a $\pm 10 \%$ energy window using a high resolution dedicated breast camera (LumaGEM 3200S/12k, Gamma Medica Ideas Inc.) constituted by a small field of view $(20 \times 15 \mathrm{~cm})$ high-resolution, solid-state semiconductor (CZT) detector mounted on a modified mammographic unit, replacing the radiographic Bucky. The camera head is composed of a pixelated (12,288 pixels) array of CZT (pixel size: $1.5 \times 1.5 \times 5 \mathrm{~mm}$ ) coupled to an array of amplifiers, the signals from which are conveyed to an electronics readout board. The system is equipped with a highly sensitive (HSEN, LEAP) long-bore, low-energy collimator (hole shape: hexagonal, hole length: $25.4 \mathrm{~mm}$, hole diameter: $2 \mathrm{~mm}$, septal thickness: $0.3 \mathrm{~mm}$ ) matched to the CZT elements. The intrinsic spatial resolution is $1.6 \mathrm{~mm}$ and the energy resolution is $<5 \%$ (average $4.6 \%$ at $140 \mathrm{Kev})$.

In all cases cranio-caudal and medio-lateral oblique projections (600 sec per view) were acquired using a 128x128 matrix size, with the breast positioned between the detector and the compression paddle of the mammographic unit to ensure a light compression of the breast parenchyma, reducing its thickness, limiting movement artefacts and improving lesion contrast. Additional breast projections could be acquired when necessary (i.e., breast bigger than the field of view, areas of increased uptake at the border of the field of view or not close to the 
Table I. Histopathological diagnosis of the 467 patients enrolled in the study: 420 with primary breast cancer and 47 with benign disease.

\begin{tabular}{lc}
\hline Diagnosis & No. of patients \\
\hline Invasive ductal carcinoma & $342^{\mathrm{a}}$ \\
Invasive lobular carcinoma & 23 \\
Mucinous carcinoma & 8 \\
Medullary carcinoma & 1 \\
Ductal carcinoma in situ (DCIS) & 42 \\
Lobular carcinoma in situ (LCIS) & 4 \\
Sclerosing adenosis & 25 \\
Fibrosis & 7 \\
Fibrosis/atypical hyperplasia & 3 \\
Fibrocystic disease & 6 \\
Fibroadenoma & 2 \\
Papillomatosis & 2 \\
Lipoma/adenosis & 1 \\
Chronic mastitis/atypical ductal & 1 \\
hyperplasia (bilateral) &
\end{tabular}

${ }^{\text {a}}$ With an associated benign disease in 7 cases (fibroadenoma in 5 cases, fibroadenomatosis in 1 case and papillomatosis in 1 case).

camera, etc.), given the flexibility of mammographic gantry in breast positioning.

The present study was performed in accordance with the Declaration of Helsinki. All patients gave their written informed consent prior to their inclusion in the study.

Histopathological diagnosis. Breast surgical specimens were fixed in $10 \%$ buffered formalin and stained with hematoxylin and eosin.

Lesions were identified according to their number and measured. The size of the carcinomas was determined according to the largest dimension and was given in millimetres. Surgical cancer specimens were sectioned in serial 5-mm slices and evaluated for tumor histological type and grading.

Tumors were categorized as invasive or in situ. According to the number of tumor foci, the carcinomas were classified as unifocal (only one focus) or multifocal/multicentric (two or more tumor foci within a single quadrant of the breast or within different quadrants of the same breast). Synchronous bilateral carcinomas were considered as separate primary tumors in the presence of a different histologic type or when there was no evidence of spread from contralateral cancer. The tumor pathologic classification (TNM) system was based on the AJCC (American Joint Commettee on Cancer) criteria according to which the index carcinoma, considered as the largest one, is used to designate $\mathrm{T}$ classification in defining multiple simultaneous invasive primary carcinomas.

Data analysis. Scintigraphic images were independently evaluated by two experienced nuclear medicine physicians who were blinded to the clinical findings, to all the other diagnostic
Table II. Pathologic tumor (pT) stage of the 420 breast cancer patients enrolled in the study.

\begin{tabular}{cc}
\hline pT classification & No. of patients \\
\hline Tis & 46 \\
T1a & 19 \\
T1b & 73 \\
T1c & 216 \\
T2 & 66 \\
\hline
\end{tabular}

Tis, tumor in situ; T1a, $\leq 5 \mathrm{~mm}$; T1b, $>0.5 \leq 1 \mathrm{~cm}$; T1c, $>1 \leq 2 \mathrm{~cm}$; $\mathrm{T} 2,>2 \leq 5 \mathrm{~cm}$.

imaging procedures data and to the final histopathological diagnoses. Scintigraphy was considered suggestive of malignancy in the presence of moderate to intense focal uptake with well-delineated contours (7).

A patient by patient comparative evaluation between breast scintigraphy and mammography findings was performed, according to Mx BI-RADS categories classification. Both scintigraphic and mammographic data were related to the histopathological findings obtained from surgical samples. The incremental value of breast scintigraphy versus $\mathrm{Mx}$ was also calculated.

Statistical analysis. Breast scintigraphy results were classified as true-positive, true-negative, false-positive or false-negative considering histology as the gold standard. Sensitivity and specificity values were then calculated. $\chi^{2}$ test was used to assess the statistical differences in sensitivity of breast scintigraphy in the carcinomas subdivided according to size ( $\leq 10$ vs $>10 \mathrm{~mm}$ carcinomas). The results were considered significant when $\mathrm{p}<0.05$. Positive predictive value (true-positives/true-positives + false-positives) of breast scintigraphy in patients with BI-RADS category 4 mammography findings was also calculated.

\section{Results}

${ }^{99 \mathrm{~m}} \mathrm{Tc}$-tetrofosmin BSGC scintigraphy was true-positive in 408/420 (97.1\%) breast cancer patients, revealing multifocal/ multicentric disease in $43 / 48(89.6 \%)$ cases and bilateral disease in $8 / 8(100 \%)$ cases. The procedure detected $480 / 499(96.2 \%)$ of the overall tumor foci, including 438 of the 453 (96.7\%) invasive carcinomas and 42 of the $46(91.3 \%)$ carcinomas in situ; according to size, it identified 130/142 (91.5\%) $\leq 10 \mathrm{~mm}$ invasive carcinomas and 308 of the $311(99 \%)>10 \mathrm{~mm}(\mathrm{p}<0.005)$. The smallest invasive carcinoma detected at breast scintigraphy measured $1.8 \mathrm{~mm}$. The results of scintigraphy in relationship with the Mx findings (BI-RADS categories) are illustrated in Table III.

Three hundred and seventy-seven $/ 408$ breast cancer patients true-positive at scintigraphy were also true-positive at $\mathrm{Mx}$ (BI-RADS categories 4 or 5) that was concordant with scintigraphy in assessing the index tumor. However, breast scintigraphy evidenced a more extensive disease in respect of $\mathrm{Mx}$ in 77 of these 377 patients ( 13 belonging to BI-RADS category 4 and 64 
Table III. BSGC scintigraphy results in relationship with mammography (Mx) findings in the 467 patients enrolled in the study.

\begin{tabular}{|c|c|c|c|}
\hline \multicolumn{2}{|c|}{ Breast scintigraphy } & \multicolumn{2}{|l|}{$\mathrm{Mx}$} \\
\hline $\begin{array}{l}\text { Type of } \\
\text { results }\end{array}$ & $\begin{array}{l}\text { No. of } \\
\text { patients }\end{array}$ & Findings & $\begin{array}{l}\text { No. of } \\
\text { patients }\end{array}$ \\
\hline \multirow[t]{4}{*}{ True-positive } & 408 & $\begin{array}{l}\text { Negative } \\
\text { (BI-RADS category 1) }\end{array}$ & 29 \\
\hline & & $\begin{array}{l}\text { Probably benign } \\
\text { (BI-RADS category } 3 \text { ) }\end{array}$ & 2 \\
\hline & & $\begin{array}{l}\text { Suspicious } \\
\text { (BI-RADS category 4) }\end{array}$ & 103 \\
\hline & & $\begin{array}{l}\text { Highly suspicious } \\
\text { (BI-RADS category 5) }\end{array}$ & 274 \\
\hline \multirow[t]{4}{*}{ False-negative } & 12 & $\begin{array}{l}\text { Negative } \\
\text { (BI-RADS category 1) }\end{array}$ & 2 \\
\hline & & $\begin{array}{l}\text { Probably benign } \\
\text { (BI-RADS category } 3 \text { ) }\end{array}$ & 1 \\
\hline & & $\begin{array}{l}\text { Suspicious } \\
\text { (BI-RADS category 4) }\end{array}$ & 4 \\
\hline & & $\begin{array}{l}\text { Highly suspicious } \\
\text { (BI-RADS category 5) }\end{array}$ & 5 \\
\hline \multirow[t]{2}{*}{ True-negative } & 40 & $\begin{array}{l}\text { Negative } \\
\text { (Bi-RADS category } 1 \text { ) }\end{array}$ & 7 \\
\hline & & $\begin{array}{l}\text { Suspicious } \\
\text { (BI-RADS category 4) }\end{array}$ & 33 \\
\hline False-positive & 7 & $\begin{array}{l}\text { Suspicious } \\
\text { (BI-RADS category 4) }\end{array}$ & 7 \\
\hline
\end{tabular}

to BI-RADS category 5), identifying the in situ component sited around invasive cancers (Fig. 1) in 56/77 cases and new tumor foci in further $21 / 77$ cases, as confirmed at surgery; these new 21 foci, all clinically occult, were ipsilateral in 16 cases with surgically proven multifocal/multicentric invasive ductal carcinomas (Fig. 2) and contralateral in 5 cases, as confirmed at surgery. Scintigraphy changed surgical management in 14 of these 77 cases (18.2\%). The remaining 31/408 breast cancer patients true-positive at scintigraphy were false-negative at $\mathrm{Mx}$ (BI-RADS category 1 or 3; Fig. 3): 26 of the 31 patients had one invasive ductal carcinoma each in heterogeneously/high dense breast ( $>10 \mathrm{~mm}$ in 7 cases and $\leq 10 \mathrm{~mm}$ in 19), 4 had one carcinoma in situ (2 DCIS in Paget's disease, 1 multicentric DCIS and 1 multicentric LCIS) and 1 had one invasive lobular carcinoma (18 $\mathrm{mm}$ in size) in association with a component in situ.

Scintigraphy was false-negative in 12 breast cancer patients, globally missing 16 tumor foci; 10 of these 12 patients had a unifocal carcinoma each (6 subcentimetric, 5-7 mm in size, invasive ductal carcinomas sited in internal quadrants, including
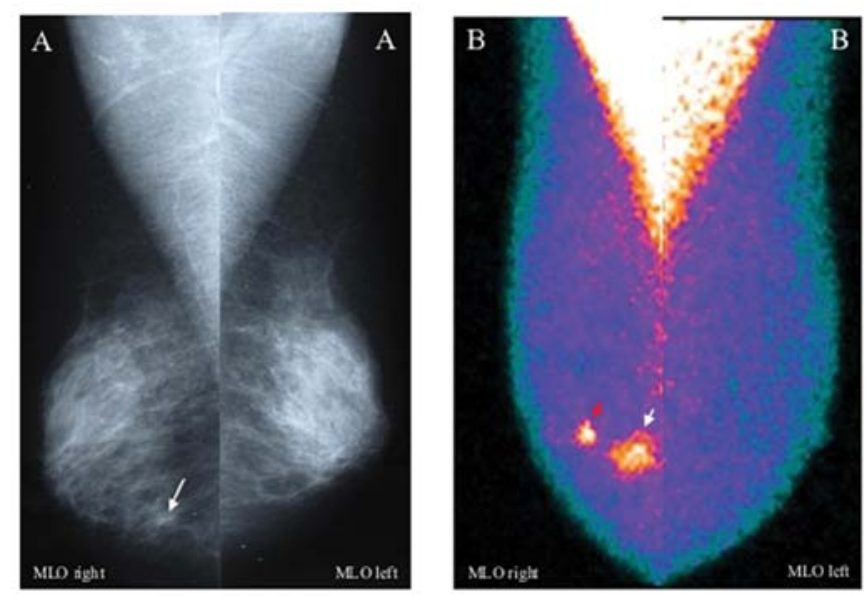

Figure 1. A 58-year-old breast cancer patient with an invasive ductal carcinoma $(13 \mathrm{~mm})$ situated in the internal lower quadrant of the right breast with an associated intraductal component. The primary tumor (white arrow) was evident at both Mx (A) and BSGC scintigraphy (B), while the intraductal component (red arrow) was evident only at scintigraphy which changed the local staging.
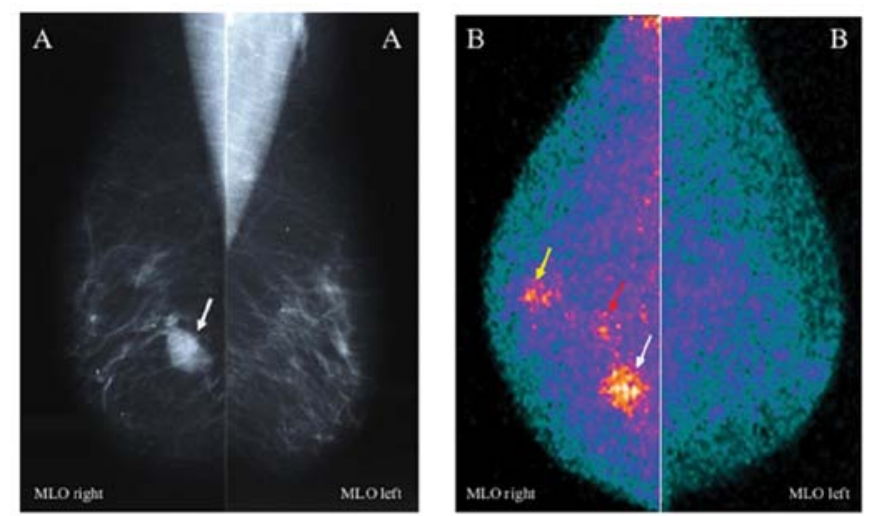

Figure 2. A 62-year-old breast cancer patient with a multicentric invasive mucinous carcinoma and an associated intraductal component in the right breast. Mx (A) only evidenced the index invasive focus (white arrow), which measured 17 , while breast scintigraphy (B) also detected the intraductal component around the index tumor (red arrow) and an additional subcentimetric $(5 \mathrm{~mm})$ invasive focus (yellow arrow). The surgical management changed from local surgery to mastectomy.
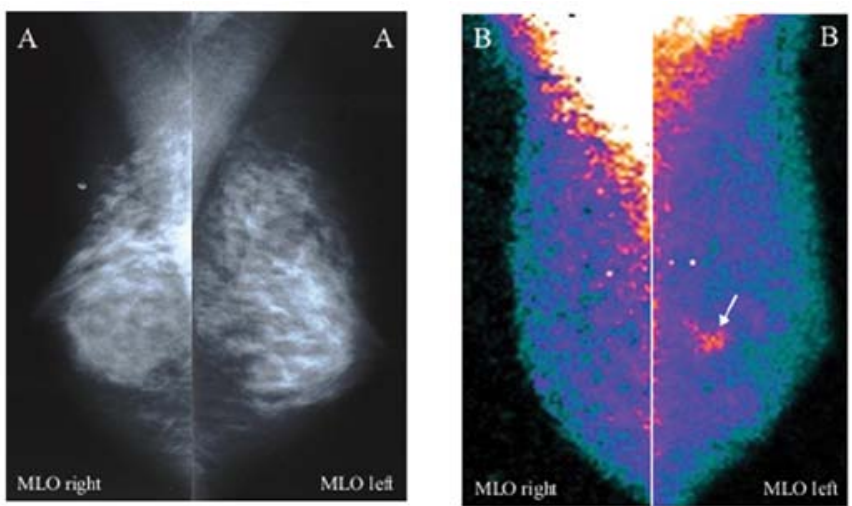

Figure 3. A 50-year-old breast cancer patient with a subcentimetric $(4 \mathrm{~mm})$ invasive ductal carcinoma between the internal quadrants of the left breast, missed at Mx (A), which was heterogeneously dense, and evidenced (arrow) at BSGC scintigraphy (B). 
2 carcinomas that was impossible to include in the field of view of the device since sited in internal upper quadrant close to the chest wall, 1 LCIS and 3 DCIS), while the remaining 2/12 patients had multicentric carcinomas (an invasive ductal with 3 subcentimetric foci, $4-5 \mathrm{~mm}$ in size, in one case and an invasive mucinous with 3 foci, 11-14 $\mathrm{mm}$ in size, in the other case).

Scintigraphy also missed 3 additional tumor foci (3-5 mm in size) in further 3 patients with multicentric invasive carcinoma in whom it identified only the index tumor, failing the diagnosis of multicentricity.

Three of the 12 breast cancer patients false-negative at scintigraphy were also false-negative at $\mathrm{Mx}$ (BI-RADS categories 1 or 3 ) and included the 2 patients with a subcentimetric invasive ductal carcinoma each sited in internal upper quadrant and excluded from the field of view of the mammogram and the patient with LCIS. The remaining $9 / 12$ breast cancer patients false-negative at scintigraphy were true-positive at $\mathrm{Mx}$.

Scintigraphy was true-negative in $40 / 47$ (85.1\%) patients with benign disease and in $47 / 55(85.4 \%)$ lesions. Of the 40 patients with benign lesions true-negative at scintigraphy, 7 were also true-negative at Mx (BI-RADS category 1), while 33 were false-positive (BI-RADS category 4). Scintigraphy was false-positive, concordantly with $\mathrm{Mx}$, in 7 patients: 4 with sclerosing adenosis, 1 with bilateral mixed pattern of chronic mastitis and atypical ductal hyperplasia, 1 with sclerohyaline fibrosis and 1 with a mixed pattern of lipoma and adenosis. Finally, of the 7 further benign lesions ascertained at surgery in 7 patients with cancer, 5 fibroadenomas and 1 fibroadenomatosis resulted negative both at scintigraphy and at $\mathrm{Mx}$, while one papillomatosis was true negative at scintigraphy and false-positive at Mx.

Globally, scintigraphy gave an additional value in respect of Mx in 141/467 overall patients (30.2\%) prospectively studied, 108 of whom with breast cancer and 33 with benign lesions, as cited above.

Finally, according to scintigraphy data and final histopathological findings relative to the 147 patients with BI-RADS category 4 mammographic pattern, breast biopsy would have been avoided in 33 of these cases (22.4\%). Negative predictive value of scintigraphy in patients with BI-RADS category 4 lesions was $82.5 \%$.

\section{Discussion}

In the present study, ${ }^{99 \mathrm{~m}} \mathrm{Tc}$-tetrofosmin breast scintigraphy acquired with a BSGC proved a very highly accurate diagnostic tool in the detection of primary breast cancer. In agreement with previous studies (8-10), the procedure demonstrated a very high sensitivity, even in the identification of carcinomas at a very early stage, such as clinically occult or subcentimetric invasive carcinomas and carcinomas in situ, while maintaining a high specificity. Moreover, when compared with $\mathrm{Mx}$, scintigraphy had an incremental value in more than one third of cases with the improvement of both sensitivity and specificity values, partially overcoming the limitations of $\mathrm{Mx}$.

In particular, in our series breast scintigraphy detected 26 primary breast carcinomas of small size majority all missed at Mx (BI-RADS category 1) for dense breast. Moreover, scintigraphy proved more accurate than $\mathrm{Mx}$ in the preoperative assessment of local disease extension, detecting a higher number of occult additional tumor foci ipsilateral or contralateral to the proven index cancer in respect of the findings ascertained at Mx; scintigraphy also proved more suitable than Mx in the identification of intraductal tumors sited around invasive carcinomas, thus changing the local staging. The majority of our patients downstaged at Mx belonged to BI-RADS 5, a category for which surgery is indicated; in our series, scintigraphy gave a more accurate local disease staging in respect of Mx in 64/279 (22.9\%) of patients with BI-RADS 5, and changed the surgical management, from breast-conservation to more radical surgery, in $18 \%$ of overall breast cancer patients. On the basis of these results, and even more in the era of breast-conservation therapy, scintigraphy acquired with a BSGC should be employed in addition to Mx in patients scheduled to surgery, since it may contribute to achieve a clearer margin excision and to complete surgical treatment in a higher number of cases, thus reducing the cases to submit to second operations and avoiding the risk of local and distant recurrences.

Scintigraphy also demonstrated a high specificity, excluding malignancy in 33 patients with suspicious findings at $\mathrm{Mx}$ (BI-RADS 4), and demonstrated a very high negative predictive value $(82.5 \%)$ in this category of patients, thus suggesting that the preoperative addition of breast scintigraphy to Mx should permit a better selection of patients to submit to biopsy.

In recent years, dynamic breast magnetic resonance imaging (MRI) is playing a growing role as a useful complementary tool to $\mathrm{Mx}$ in both screening and diagnosis of primary breast cancer as well as in the preoperative staging of the affected breast in women with newly diagnosed primary breast cancer given its very high sensitivity and its independence of breast density (12-14). MRI has also been recently recommended instead of Mx by the American Cancer Society (15) in screening high-risk patients with mammographically dense breasts.

BSGC scintigraphy has demonstrated its usefulness in detecting small size $(<1 \mathrm{~cm})$ non palpable carcinomas occult at $\mathrm{Mx}$ in women at high risk for breast cancer (16), but no comparative studies between BSGC scintigraphy and MRI testing their sensitivity and specificity have been carried out in patients screened for breast cancer. However, these two procedures have been compared in the diagnosis of primary breast cancer showing similarly high sensitivity values (89\% for breast scintigraphy and $100 \%$ for MRI), while specificity was markedly higher for breast scintigraphy (71 vs 25\%) (17). BSGC scintigraphy and MRI have also been compared in a series of patients with newly ascertained primary breast cancer scheduled to surgery, achieving a correct preoperative assessment of local disease extension in a high percentage of cases ( 88.5 and $80 \%$, respectively), but MRI has lead to an overstaging in a higher number of cases than scintigraphy (18). The risk of surgically overtreating patients on the basis of MRI findings thus seems to exist, as reported by other authors (13). MRI presents other drawbacks such as high cost, time-consuming and variability in technique and interpretation criteria, and it requires very experienced radiologists. Moreover, the procedure is influenced by menstrual phase and it is also contraindicated in selected patients (i.e., those with allergy to contrast medium and with obesity, pacemakers, aneurysm clips or severe claustrophobia). Thus, BSGC scintigraphy could represent an alternative to MRI in selected cases, as also suggested by the 
SNM practice guidelines (7). Moreover, BSGC scintigraphy is relatively less expensive (3-5 times less than MRI), simple to perform and well tolerated by the patients, and gives very high resolution images, easy and quick to read and well comparable with mammographic findings. However, also this scintigraphic procedure presents some limitations, such as radiation exposure that could limit its routine use, especially in screening programs. Tumor size seemed to represent the most important factor affecting the performance of scintigraphy, since the majority of false-negative findings were related to subcentimetric carcinomas. Moreover, lesion site may also affect sensitivity, since a high percentage of small tumors negative at breast scintigraphy in our series were located in internal quadrants or were excluded from the field of view of the device as happened in two cases. The importance of patient positioning in acquiring breast scintigraphy with dedicated devices should be always taken into account to avoid patient misplacement. In our series, the procedure also missed three large carcinomas, probably due to their low cellularity and slow proliferative growth, the histological types being mucinous or lobular. Recent advances in technology could further increase the performance of BSGC scintigraphy used in the present study due to the development of dual-head systems which simultaneously acquire opposing breast views, thus reducing lesion distance to detector and increasing the sensitivity especially in the detection of small size lesions. These systems also improve collimation providing a higher number of counts per pixel in scintigraphic images thus permitting to reduce the radiotracer dose without affecting the quality of images.

A larger clinical application of BSGC scintigraphy is thus suggested although many prospective trials are needed, particularly with the aim of identifying those subgroups of patients who would more benefit from scintigraphy employment. In the era of breast-conservation surgery, the impact of breast scintigraphy on changing the therapeutic approach in cases with additional foci detected only by this procedure should also be further analyzed in terms of improvement in surgical care and prognosis. However, cost-benefit analysis is needed to justify the use of BSGC scintigraphy in combination with conventional diagnostic imaging methods as an adjunctive tool.

Another molecular breast imaging has been recently tested in primary breast cancer patients as ${ }^{18} \mathrm{~F}$-fluorodeoxyglucose (FDG) positron emission mammography (PEM) which, like BSGC scintigraphy, has also showed high sensitivity and good specificity $(19,20)$. However, no comparative studies between the two procedures have been carried out in the same patient population. The detectors employed for both procedures present very similar spatial resolution; differences in performance thus could possibly be due to the different characteristics of the radiotracers rather than the technology.

In conclusion, breast scintigraphy acquired with a BSGC proved a highly sensitive diagnostic tool, even in small size carcinoma detection, while maintaining a high specificity. In our series, this procedure increased both the sensitivity of $\mathrm{Mx}$, especially in dense breast and in multifocal/multicentric disease, and the specificity as well as it better defined local tumor extension, thus guiding the surgeon to a more appropriate surgical treatment. A wider employment of this procedure is suggested as a complementary tool to $\mathrm{Mx}$.

\section{Acknowledgements}

The study was partly funded by the Fondazione Banco di Sardegna.

\section{References}

1. Kolb TM, Lichy J and Newhouse JH: Comparison of the performance of screening mammography, physical examination, and breast US and evaluation of factors that influence them: an analysis of 27,825 patient evaluations. Radiology 225: $165-175,2002$.

2. Lagios MD, Westdahal PR and Rose MR: The concept and implications of multicentricity in breast carcinoma. In: Pathology Annula. Sommers S and Rosen P (eds). Appleton-Century-Crofts, New York, NY, 1981.

3. Holland R, Veling SHJ, Mravunac M and Hendriks JHCL: Histologic multifocality of Tis, T1-2 breast carcinomas: implications for clinical trials of breast-conserving surgery. Cancer 56 : 979-990, 1985

4. Liberman M, Sampalis F, Mulder DS and Sampalis JS: Breast cancer diagnosis by scintimammography: a meta-analysis and review of the literature. Breast Cancer Res Treat 80: 115-126, 2003.

5. Howarth D, Sillar R, Clark D and Lan L: Technetium-99m sestamibi scintimammography: the influence of histopathological characteristics, lesion size and the presence of carcinoma in situ in the detection of breast carcinoma. Eur J Nucl Med 26: 1475-1481, 1999.

6. Khalkhali I, Villanueva-Meyer J, Edell SL, Connolly JL, Schnitt SJ, Baum JK, Houlihan MJ, Jenkins RM and Haber SB: Diagnostic accuracy of ${ }^{99 \mathrm{~m}} \mathrm{Tc}-$ Sestamibi breast imaging: multicenter trial results. J Nucl Med 41: 1973-1979, 2000.

7. Goldsmith SJ,Parson W, Guiberteau MJ, Stern LH,Lanzkowsky L, Weigert J, Heston TF, Jones E, Buscombe J and Stabin MG: SNM practice guideline for breast scintigraphy with breast specific $\gamma$-cameras 1.0. J Nucl Med Technol 38: 219-224, 2010.

8. Rhodes DJ, O'Connor MK, Phillips SW, Smith RL and Collins DA: Molecular breast imaging: a new technique using technetiumTc scintimammography to detect small tumors of the breast. Mayo Clin Proc 80: 24-30, 2005.

9. Spanu A, Cottu P, Manca A, Chessa F, Sanna D and Madeddu G: Scintimammography with dedicated breast camera in unifocal and multifocal/multicentric primary breast cancer detection: a comparative study with SPECT. Int J Oncol 31: 369-377, 2007.

10. Spanu A, Chessa F, Meloni GB, Sanna D, Cottu P, Manca A, Nuvoli $S$ and Madeddu G: The role of planar scintimamography with high-resolution dedicated breast camera in the diagnosis of primary breast cancer. Clin Nucl Med 33: 739-742, 2008.

11. D'Orsi CJ and Kopans DB: Mammographic features analysis. Semin Roentgenol 18: 204-230, 1993.

12. Pediconi F, Catalano C, Padula S, Roselli A, Moriconi E, Pronio AM, Kirchin MA and Passariello R: Contrast-enhanced magnetic resonance mammography: does it affect surgical decision-making in patients with breast cancer? Breast Cancer Res Treat 106: 65-74, 2007.

13. Drew PJ, Chatterjee S, Turnbull LW, Read J, Carleton PJ, Fox JN, Monson JR and Kerin MJ: Dynamic contrast enhanced magnetic resonance imaging of the breasts is superior to triple assessment for the pre-operative detection of multifocal breast cancer. Ann Surg Oncol 6: 599-603, 1999.

14. Houssami N and Hayes DF: Review of preoperative magnetic resonance imaging (MRI) in breast cancer: should MRI be performed on all women with newly diagnosed, early stage breast cancer? CA Cancer J Clin 59: 290-302, 2009.

15. Saslow D, Boetes C, Burke W, Harms S, Leach MO, Lehman CD, Morris E, Pisano E, Schnall M, Sener S, Smith RA, Warner E, Yaffe M, Andrew KS and Russel CA: American Cancer Society guidelines for breast screening with MRI as an adjunct to mammography. CA Cancer J Clin 57: 75-89, 2007.

16. Brem RF, Schoonjans JM, Kieper DA, Majewski S, Goodman S and Civelek C: High-resolution scintimammography: a pilot study. J Nucl Med 43: 909-915, 2002.

17. Brem RF, Petrovitch I, Rapelyea JA, Young H, Teal C and Kelly T: Breast-specific gamma camera imaging with ${ }^{99 \mathrm{~m}} \mathrm{Tc}$-sestamibi and magnetic resonance imaging in the diagnosis of breast cancer: a comparative study. Breast J 13: 465-469, 2007. 
18. Spanu A, Chessa F, Sanna D, GB Meloni, D Sanna, Cottu P, Nuvoli S and Madeddu G: ${ }^{99 \mathrm{~m} T c-t e t r o f o s m i n ~ M o l e c u l a r ~ B r e a s t ~}$ Imaging (MBI) with high resolution dedicated breast camera (DBC) and breast dynamic Magnetic Resonance Imaging (MRI) in the preoperative staging of breast cancer: a comparative study. Eur J Nucl Med Mol Imaging 36 (Suppl. 2): S471, 2009.

19. Berg WA, Weinberg IN, Narayanan D, Lobrano ME, Ross E, Amodei L, Tafra L, Adler LP, Uddo J, Stein W III and Levine EA: High-resolution fluorodeoxyglucose positron emission tomography with compression (positron emission mammography) is highly accurate in depicting primary breast cancer. Breast J 12: 309-323, 2006.
20. Schilling K, Narayanan D, Kalinyak JE, The J, Velasquez MV, Kahn S, Saady M, Mahal R and Chrystal L: Positron emission mammography in breast cancer presurgical planning: comparison with magnetic resonance imaging. Eur J Nucl Med Mol Imaging 38: 23-36, 2011. 